\title{
Peripheral capillary non-perfusion in Birdshot retinochoroiditis: a novel finding on ultra- widefield fluorescein angiography
}

\author{
Ilaria Testi ${ }^{1}$, Sofia Ajamil-Rodanes ${ }^{1}$, Abdulrahman F. AlBloushi ${ }^{1}$, Carlos Pavesio ${ }^{1}$ \\ ${ }^{1}$ Moorfields Eye Hospital, National Health Service Foundation Trust, London, United Kingdom
}

\author{
Corresponding Author \\ Carlos Pavesio \\ Moorfields Eye Hospital \\ 162 City Rd, Old Street, London EC1V 2PD \\ c.pavesio@nhs.net
}

\begin{abstract}
Purpose To report a novel peripheral finding in patients with Birdshot retinochoroiditis (BRC) using ultra-widefield fluorescein angiography (UWFFA).

Methods UWFFA images from 90 patients diagnosed with BRC were reviewed. All images were evaluated for the presence of peripheral retinal ischemic changes and ischemia-related complications.

Findings Thirty-six eyes of 24 patients showed features of peripheral ischemia, including 18 eyes with peripheral capillary non-perfusion (CNP), 5 eyes with teleangectasias, with or without microaneurysms, and 13 eyes with both teleangectasias and CNP. None of the 36 eyes with peripheral ischemic changes developed retinal neovascularization following the diagnosis of peripheral ischemia.
\end{abstract}

Conclusions Peripheral ischemia may present in eyes with chronic inflammation as seen in BRC but does not seem to progress to neovascular complications. This is likely to be more common than suspected and has now become evident following the advent of UWFFA.

Keywords Birdshot retinochoroiditis; ultra-widefield fluorescein angiography; peripheral capillary non-perfusion; teleangectasias; retinal neovascularization. 


\section{Introduction}

Birdshot retinochoroiditis $(B R C)$ is a rare form of chronic, progressive, sight-threatening uveitis, predominant in the middle-aged Caucasian population with female predominance. ${ }^{1}$ Characterized by both retinal inflammation and stromal choroiditis, the disease manifests with a distinctive clinical phenotype, involving bilateral, creamy, ovoid, choroidal lesions, giving the typical 'Birdshot' appearance. ${ }^{1,2}$ The strong association with the human leukocyte antigen (HLA)-A29, reported in virtually $100 \%$ of the affected patients, suggests the role of an autoinflammatory response elicited by environmental factors in a genetically predisposed individual. ${ }^{3}$ Symptoms include floaters, blurred vision, photopsias and nyctalopia, although visual acuity is often preserved, at least in the early stages. Imaging techniques, such as fundus fluorescein angiography (FFA), indocyanine green angiography (ICGA), fundus autofluorescence, spectral-domain optical coherence tomography (OCT) and the more recent enhanced depth imaging OCT, are currently used for the diagnosis and management of the disease. The multimodal imaging approach is useful in detecting the presence of active vasculitis and inflammatory choroidal lesions, and to evaluate changes in retinal structures, subretinal fluid accumulation, and retinal and choroidal thickness. ${ }^{1,4,5}$

Advanced ultra-widefield (UWF) technologies have been recently applied in the field of ocular inflammatory disorders, offering a step forward in retinal imaging with clear advantages over standard multi-field images. UWF fluorescein angiography (UWFFA) allows simultaneous angiographic images of mid and peripheral retina up to a 200-degree field of view in a single frame. Several studies described the potential benefits of UWF in the management of uveitis. UWFFA is advantageous in detecting peripheral active vasculitis, areas of capillary non-perfusion (CNP), retinal neovascularization and inflammatory lesions. ${ }^{5-12}$ The additional information provided by this technique has influenced disease management and aid in making therapeutic decisions. ${ }^{11}$

Several publications have focused on UWF angiographic features of occlusive retinal vasculitis, while there is no literature on $\mathrm{BRC} .^{7,13-16}$ Here we report peripheral retinal ischemic changes in patients with Birdshot-related vasculitis, not associated with the development of retinal 
neovascularization and ischemia-related complications. To the best of our knowledge, such angiographic features have not been published previously.

\section{Methods}

UWF imaging studies of patients diagnosed with BRC who were seen at the uveitis clinic at Moorfields Eye Hospital between January 2012 and December 2018 with a minimum follow up of 12 months were reviewed. Diagnostic criteria of BRC were bilateral involvement of the disease with retinal vasculitis and choroidal inflammatory disease, which could be clinically obvious as the typical Birdshot lesions, or detected by ICGA, and most importantly by a positive HLA-A29 test. ${ }^{3}$ Exclusion criteria were multiple keratic precipitates, posterior synechiae and the presence of infections, neoplasms or other inflammatory diseases causing multifocal choroidal lesions. ${ }^{17}$ All UWFFA images were acquired at baseline with the Optos 200Tx ultra-widefield retinal imaging system (Optos PLC, Dunfermline, Scotland, UK), which can image up to 200-degree of the retina in a single view. All UWFFA images were evaluated by two independent assessors for the presence of peripheral CNP, telangiectasias, microaneurysms and ischemia-induced neovascularisation. A third independent assessor graded the images in case of disagreement.

\section{Results}

Ninety patients diagnosed with BRC underwent Optos 200Tx UWFFA at baseline and were included in the study. Detected fluorangiographic findings previously described in BRC included early hypofluorescent lesions with subtle late staining, diffuse and focal retinal vascular leakage, optic nerve leakage and macular leakage. In addition to the previously reported angiographic features, we describe a total of 36 eyes from 24 patients with features of peripheral ischemia. The UWFFA signs included 18 eyes from 12 patients with peripheral CNP (Figure 1), 5 eyes from 4 patients with peripheral teleangectasias (Figure 2), with or without microaneurysms, and 13 eyes from 10 patients with both peripheral teleangectasias and CNP (Figure 3). All areas of CNP did not involve more than 1 quadrant at baseline. Areas of ischemia were localised to the peripheral area in 30 eyes, extended to the mid-peripheral area in 6 eyes, not involving the posterior pole. None of the 36 eyes with 
peripheral ischemic changes developed retinal neovascularization following the diagnosis of peripheral ischemia. Median follow-up was 26 months (range: 12-72).

Mean age of patients with peripheral CNP was 54.5 years (range $43-64$ years). Any statistically significant difference was found between them and the patients with no ischaemic findings (mean age 52.0 years [range $37-66$ years], $p>0.05)$.

\section{Discussion}

UWFFA is considered to be an extremely useful tool to diagnose and monitor inflammatory diseases affecting the posterior segment of the eye. Several publications have focused on the UWF angiographic features of occlusive retinal vasculitis, reporting vascular wall staining, extravascular leakage, areas of retinal ischaemia and non-perfusion and retinal neovascularization. ${ }^{7,10,13-15,17}$ We describe peripheral ischemic findings in patients with Birdshot-related retinal vasculitis. The results of the current study highlight how peripheral vascular occlusion and inflammation-induced retinal ischemia can occur in inflammatory disorders that are not typically occlusive in nature. Among the patients included in the study, $24.6 \%$ revealed angiographic features at baseline consistent with peripheral retinal ischemic changes, including increased ramifications and straightening of peripheral vessels, teleangectasias, microaneurysms and areas of CNP.

Inflammation-induced vascular occlusion and ischemia are known to cause retinal neovascularisation, with complications such as recurrent vitreous hemorrhages, traction retinal detachment, rubeosis iridis and neovascular glaucoma, potentially leading to functional visual loss. None of the patients included in the study developed retinal neovascularization and other ischemiarelated sequelae or received laser treatment following the diagnosis of peripheral retinal ischemia. Considering that the risk of neovessels formation is significantly increased in eyes with retinal ischemia involving $\geq 2$ quadrants, and that the areas of CNP detected in Birdshot patients at baseline involved no more than 1 quadrant, we suggest no need for laser photocoagulation treatment of the affected areas. ${ }^{18,19}$

\section{Conclusion}


Peripheral ischaemia may present in eyes with chronic inflammation as seen in BRC but does not seem to progress to neovascular complications. This is likely to be more common than suspected and has now become evident following the advent of UWFFA.

Limitations of the study is the retrospective method, which leads to a lack of standardization in documentation and hence missing data, including the correlation between peripheral CNP and functional measures, such as visual acuity, contrast sensitivity, colour vision, visual field and electrophysiology testing, and with underlying diseases, including diabetes, systemic hypertension or hyperlipidaemia. Future prospective studies based on standardized protocols for acquisition of images and clinical parameters can be useful to address potential correlations between CNP areas and clinical findings, and to compare clinical and angiographic findings in Birdshot patients with and without peripheral ischemia.

Figures legend 
Figure 1 Ultra-wide field fluorescein angiography in two cases of active Birdshot retinochoroiditis showing retinal vascular leakage and optic disc hyperfluorescence. Areas of capillary non perfusion are detectable in the peripheral temporal (A) and superior-nasal (B) retina, with increased ramifications of peripheral vessels.

Figure 2 Ultra-wide field fluorescein angiography in a case of active Birdshot retinochoroiditis showing diffuse retinal vascular leakage, optic disc hyperfluorescence and teleangectasias both in the peripheral temporal (better seen in the magnified square 1) and nasal retina.

Figure 3 Ultra-wide field fluorescein angiography in three cases of active Birdshot retinochoroiditis showing peripheral capillary non-perfusion and teleangectasias in the peripheral temporal retina (better seen in the magnified squares $1,2,3)$, with straightening of peripheral vessels $(A, B, C)$. 
Funding This work was supported by the National Institute for Health Research (NIHR) Biomedical Research Centre based at Moorfields Eye Hospital NHS Foundation Trust and UCL Institute of Ophthalmology. The views expressed are those of the authors and not necessarily those of the NHS, the NIHR or the Department of Health.

Availability of data and materials All the data and images pertaining to the cases are available with the corresponding author.

Authors' contributions IT, SA and AA performed data collection and analysis, and contributed to literature review and preparation of the manuscript. CP provided the concept and design, intellectual content and critical review of the manuscript. All authors read and approved the final manuscript.

Competing interests The authors have no relevant affiliations or financial involvement with any organization or entity with a financial interest in or financial conflict with the subject matter or materials discussed in the manuscript. This includes employment, consultancies, honoraria, stock ownership or options, expert testimony, grants or patents received or pending, or royalties.

Consent An informed consent was taken from patients.

\section{Abbreviations}

HLA-A29 Human leukocyte antigen - A29

FFA Fundus fluorescein angiography

OCT Optical coherence tomography

ICGA Indocyanine green angiography

UWF Ultra-wide field

UWFFA Ultra-wide field fluorescein angiography

CNP Capillary non-perfusion

\section{BIBLIOGRAPHY}

1. Minos E, Barry RJ, Southworth S, et al. Birdshot chorioretinopathy: current knowledge and 
new concepts in patho- physiology, diagnosis, monitoring and treatment. Orphanet $\mathrm{J}$ Rare Dis. 2016;11:61.

2. Shah KH, Levinson RD, Yu F, Goldhardt R, Gordon LK, Gonzales CR, et al. Birdshot chorioretinopathy. Surv Ophthalmol 2005;50:519-541.

3. Herbort CP Jr, Pavesio C, LeHoang $P$, et al. Why birdshot retinochoroiditis should rather be called 'HLA-A29 uveitis'?. Br J Ophthalmol. 2017;101(7):851-855.

4. Pohlmann D, Macedo S, Stübiger N, Pleyer U, Joussen AM, Winterhalter S. Multimodal Imaging in Birdshot Retinochoroiditis. Ocul Immunol Inflamm. 2017;25(5):621-632.

5. Teussink MM, Huis In Het Veld PI, de Vries LA, Hoyng CB, Klevering BJ, Theelen T. Multimodal imaging of the disease progression of birdshot chorioretinopathy. Acta Ophthalmol. 2016;94(8):815-823.

6. Aggarwal K, Mulkutkar S, Mahajan S, et al. Role of Ultra-Wide Field Imaging in the Management of Tubercular Posterior Uveitis. Ocul Immunol Inflamm. 2016;24(6):631-636.

7. Jones NP, Sala-Puigdollers A, Stanga PE. Ultra-widefield fundus fluorescein angiography in the diagnosis and management of retinal vasculitis. Eye (Lond). 2017;31(11):1546-1549.

8. Karampelas M, Sim DA, Chu C, et al. Quantitative analysis of peripheral vasculitis, ischemia, and vascular leakage in uveitis using ultra-widefield fluorescein angiography. Am J Ophthalmol. 2015;159(6):1161-1168.e1.

9. Kim P, Sun HJ, Ham DI. Ultra-wide-field angiography findings in acute Vogt-KoyanagiHarada disease. Br J Ophthalmol. 2019;103(7):942-948.

10. Mesquida M, Llorenç V, Fontenla JR, Navarro MJ, Adán A. Use of ultra-wide-field retinal imaging in the management of active Behçet retinal vasculitis. Retina. 2014;34(10):2121-7.

11. Pecen PE, Petro KF, Baynes K, Ehlers JP, Lowder CY, Srivastava SK. Peripheral Findings and Retinal Vascular Leakage on Ultra-Widefield Fluorescein Angiography in Patients with Uveitis. Ophthalmol Retina. 2017;1(5):428-434.

12. Leder HA, Campbell JP, Sepah YJ, et al. Ultra-wide-field retinal imaging in the management of non-infectious retinal vasculitis. J Ophthalmic Inflamm Infect.2013;3(1):30.

13. Aggarwal K, Mulkutkar S, Mahajan S, et al. Role of ultra- wide field imaging in the management of tubercular poster- ior uveitis. Ocul Immunol Inflamm 2016;24(6):631-636.

14. Campbell JP, Beardsley RM, Palejwala NV, et al. Peripheral vascular leakage in uveitis: clinical and angiographic findings. Ophthalmology. 2015;122(6):1269-1270.

15. Agrawal R, Gunasekeran DV, Gonzalez-Lopez JJ, et al. Peripheral retinal vasculitis: analysis of 110 consecutive cases and a contemporary reappraisal of tubercular etiology. Retina 2017;37(1):112-117.

16. Levinson RD, Brézin A, Rothova A, Accorinti M, Holland GN. Research criteria for the diagnosis of birdshot chorioretinopathy: results of an international consensus conference. Am J Ophthalmol. 2006;141(1):185-7. 
17. Agarwal A, Afridi R, Agrawal R, Do DV, Gupta V, Nguyen QD. Multimodal Imaging in Retinal Vasculitis. Ocul Immunol Inflamm. 2017;25(3):424-433.

18. Rouvas A, Nikita E, Markomichelakis N, Theodossiadis P, Pharmakakis N. Idiopathic retinal vasculitis, arteriolar macroaneurysms and neuroretinitis: clinical course and treatment. $J$ Ophthalmic Inflamm Infect. 2013; 3(1):21.

19. Sharief L, Lightman S, Blum-Hareuveni T, Bar A, Tomkins-Netzer O. Clinical Outcome of Retinal Vasculitis and Predictors for Prognosis of Ischemic Retinal Vasculitis. Am J Ophthalmol. 2017;177:206-212. 